\title{
Identification and Characterization of the SSB1 Locus Involved in Symptom Development by Spring beauty latent virus Infection in Arabidopsis thaliana
}

\author{
Koki Fujisaki, Fumi Hagihara, Yoshihiro Azukawa, Masanori Kaido, Tetsuro Okuno, and Kazuyuki Mise \\ Laboratory of Plant Pathology, Graduate School of Agriculture, Kyoto University, Kyoto 606-8502, Japan \\ Submitted 1 March 2004. Accepted 28 April 2004.
}

\begin{abstract}
The natural variation of Arabidopsis thaliana in response to a bromovirus, Spring beauty latent virus (SBLV), was examined. Of 63 Arabidopsis accessions tested, all were susceptible when inoculated with SBLV, although there was a large degree of variation in symptom development. Most accessions, including Columbia (Col-0), were symptomless or developed only mild symptoms, but four accessions, including S96, showed severe symptoms of SBLV infection. Genetic analysis suggested that the difference in the responses of Col-0 and S96 to SBLV was controlled by a single semidominant locus. We have designated this locus SSB1 (symptom development by SBLV infection). By using genetic markers, SSB1 was mapped to chromosome IV. The patterns of distribution and accumulation of SBLV in sensitive accessions were similar to those in the insensitive accessions. In addition, symptom development in S96 by SBLV infection was critically interrupted by the presence of the $N a h G$ gene, which encodes salicylic acid (SA) hydroxylase. These data suggest that symptom development in A. thaliana controlled by $S S B 1$ is independent of the efficiency of SBLV multiplication and is dependent on SA signaling.
\end{abstract}

Viral diseases of plants are established under the control of many plant and viral factors, including those required for viral multiplication and symptom induction. The development of symptoms is determined by interactions between these factors. To date, however, our understanding of such plant-virus interactions remains insufficient.

Arabidopsis thaliana has been used as a model plant in many studies, including the analysis of plant-microbe interactions (Eckardt 2001; Eckardt et al. 2001). This plant species grows in diverse environments and many accessions of $A$. thaliana have diverged significantly in their susceptibility to pathogens (Alonso-Blanco and Koornneef 2000; Kover and Schaal 2002). As for plant viruses, different responses in Arabidopsis accessions have been reported (Dardick et al. 2000; Lee et al. 1994, 1996; Leisner and Howell, 1992; Martín Martín et al. 1999; Park et al. 2002; Revers et al. 2003; Simon et al. 1992). If virus infection is restricted, viruses cannot induce systemic symptoms. For instance, Beet curly top virus (BCTV) Logan strain and Turnip mosaic virus, which induce systemic symptoms in the A. thaliana accession Col-0, do not induce visible symptoms in some other accessions because

Corresponding author: Kazuyuki Mise; Telephone: +81-75-753-6132; Fax: +81-75-753-6131; E-mail: kmise@kais.kyoto-u.ac.jp their systemic spread is restricted (Lee et al. 1994; Martín Martín et al. 1999). A mechanism of conditioning virus infectivity in A. thaliana accessions has been well studied for Tobacco etch virus (TEV) infection, and the RTM1 and RTM2 genes controlling restriction of the long-distance movement of TEV have been isolated (Chisholm et al. 2000; Whitham et al. 2000). In other cases, viruses induce necrotic lesions known as the hypersensitive response (HR), which is a typical plant defense response restricted to initially infected regions (Dempsey et al. 1993; Takahashi et al. 1994). On the other hand, systemic infection of viruses does not necessarily induce symptoms. The grape strain of Tobacco ringspot virus (TRSV) systemically infects some $A$. thaliana accessions, including Col-0, without visible symptoms, which is termed 'tolerance.' TRSV induces severe symptoms in other accessions, including Estland. By using genetic analysis, the TTRl locus controlling tolerance to TRSV was identified on chromosome V (Lee et al. 1996). Similar observations have been reported using the BCTV CFH strain (Park et al. 2002). Thus, although relatively many studies have focused on resistance genes for virus multiplication in plants, poor information has been available about plant genes controlling symptom development.

Bromoviruses are a group of icosahedral plant viruses whose genome comprises positive-sense tripartite RNAs designated RNA1, RNA2, and RNA3 (Lane 1981). The bromovirus Brome mosaic virus (BMV) was the first RNA virus for which infectious in vitro transcripts were synthesized from cDNA clones of genomic RNAs (Ahlquist et al. 1984). The viral factors required for BMV multiplication have also been well characterized (Ahlquist 1999; Sullivan and Ahlquist 1997). In contrast, little is known about plant factors involved in bromovirus infection and symptom, partly because BMV shows low infectivity in A. thaliana. Recently, we have shown that another bromovirus, Spring beauty latent virus (SBLV), efficiently infects the A. thaliana accession Col-0 (Fujisaki et al. 2003a), suggesting that SBLV is a useful virus for analyzing plantbromovirus interactions by the reverse and forward genetic approach.

As a first step toward the elucidation of SBLV-A. thaliana interactions, natural variation in response to SBLV infection was examined. SBLV asymptomatically infects Col-0 (insensitive) (Fujisaki et al. 2003a), whereas it induced severe symptoms in some other A. thaliana accessions, such as S96 and Ei-2 (sensitive). Infectivity of SBLV in the tested sensitive accessions was similar to that in the insensitive one. Plant factors controlling symptom production in SBLV infection were analyzed using a genetic approach. 


\section{RESULTS}

\section{Multiple responses}

of various Arabidopsis accessions to SBLV.

A total of 63 Arabidopsis accessions were inoculated with SBLV virions at 3 to 4 weeks after sowing, and symptoms were observed at 14 days postinoculation (dpi). SBLV systemically infects the accession Col-0 but does not induce visible symptoms in this accession (Fujisaki et al. 2003a). Similarly, most of the accessions tested were also symptomless or developed only mild symptoms at 14 dpi. However, four accessions (S96, Fr-2, Ei-2, and Abd-0) infected with SBLV exhibited severe symptoms (Table 1). Inoculated leaves of these four accessions exhibited yellowing and necrotic symptoms. In S96 and Fr-2, uninoculated rosette leaves also showed yellowing and curling, and most of these leaves died by 14 dpi (Table 1, Fig. 1A). In some S96 and Fr-2 plants, newly emerging leaves did not die, but showed severe curling at 14 dpi (Fig. 1B). In addition, S96 and Fr-2 plants infected with SBLV exhibited curling of stems and cauline leaves and produced very few seeds (data not shown). In the Ei-2 and Abd-0 accessions, yellowing and necrotic symptoms on uninoculated rosette leaves were mild, in contrast to those of S96 and Fr-2, although severe stunting of uninoculated rosette leaves of $\mathrm{Ei}-2$ and Abd- 0 was observed (Fig. 1A). Ei-2 and Abd-0 are late flowering compared with S96 and Fr-2, and no bolting tissues emerged at 14 dpi, even in mock-inoculated plants. However, when older plants of Ei-2 and Abd-0 (at 6 to 7 weeks after sowing) were inoculated, curling stems were observed at 14 dpi (data not shown).

Systemic infectivity of SBLV in the 63 accessions was examined. At least 10 plants were tested for each accession. Press blot analysis of SBLV infection at $14 \mathrm{dpi}$ demonstrated that SBLV coat protein (CP) was detected in uninoculated upper tissues of all accessions (data not shown). The frequency of systemic infection with SBLV in S96, Fr-2, Ei-2, and Abd-0, in which severe symptoms were induced by SBLV infection was approximately 100, 91, 92, and 100\%, respectively. Moreover, the frequency of systemic infection with SBLV in many other accessions, including Col-0, was similar to that in the accessions sensitive to SBLV (approximately 80 to 100\%), whereas that in the Pla-0 accession was low (approximately 40\%). Together, these data indicate that susceptibility to SBLV does not correlate with sensitivity to SBLV in Arabidopsis accessions.

\section{Time-course of distribution and accumulation of SBLV in several Arabidopsis accessions.}

To examine infectivity of SBLV in detail, four accessions, Col-0, Pla-0, S96, and Ei-2, were selected on the basis of their

Table 1. Arabidopsis accessions phenotypes based on symptoms after Spring beauty latent virus (SBLV) infection

\begin{tabular}{ll}
\hline Symptom & \multicolumn{1}{c}{ Arabidopsis accession } \\
\hline No or mild symptoms & Ag-0, Ak-1, Ang-0, Bay-0, Be-0, Berkeley, \\
& Bla-2, Bs-1, Bsch-0, C24, Chi-0, Co, Col-0, \\
& Condara, Da, Db-0, Di-G, Dra-0, En-D, \\
& ENF, Er, EST, Fe-1, Fi-0, Ga-0, Gd-1, Hl-0, \\
& Hodja-Obi-Garm, Jm-0, Kl-0, Ler-0, Li-5, \\
& LIN, Litva, Mh-0, Nd-1, No-0, Nok-0, Nw- \\
& 0, Ob-0, Old-1, Oy-1, Pa-1, Petergof, Pi-0, \\
& Pla-0, RLD-1, Rubeznoe-1, Sg-1, Shahdara, \\
& Sn, Sorbo, Tol-0, Tsu-1, Tu-0, Wc-1, Wil-1, \\
& Wt-1, Wu-0 \\
Severe necrotic symptoms ${ }^{\mathrm{a}}$ & S96, Fr-2 \\
Severe stunting symptoms ${ }^{\mathrm{b}}$ & Ei-2, Abd-0 \\
\hline a S96 and Fr-2 infected with & SBLV showed severe necrotic symptoms in \\
uninoculated rosette leaves. & \\
b Ei-2 and Abd-0 infected & with SBLV showed severe stunting in \\
uninoculated rosette leaves. &
\end{tabular}

different responses to SBLV infection and were used for timecourse analysis of SBLV spread. Inoculated plants were harvested at 2, 4, and $7 \mathrm{dpi}$, and SBLV CP was detected by press blot analysis. SBLV infection in Col-0 was first detected as small dots at 2 dpi and had spread through the whole area of inoculated leaves and into some uninoculated leaves by $4 \mathrm{dpi}$. At $7 \mathrm{dpi}$, almost all plant tissues were infected with SBLV (Fig. 2). However, in the inoculated leaves of Pla-0, SBLV infection was observed as small dots even at $4 \mathrm{dpi}$, indicating that at least local spread of SBLV is delayed in Pla-0. On the other hand, the SBLV-sensitive accessions S96 and Ei-2 showed a distribution pattern of SBLV CP similar to that of Col-0 (Fig. 2).

We then checked the time-course accumulation of SBLV CP in Col-0, S96, and Ei-2 plants. The accumulation patterns of SBLV CP in inoculated leaves of Col-0 and Ei-2 were similar. Accumulation of SBLV CP in S96 was delayed to some degree at 1 and 2 dpi but reached similar levels in the three accessions at $3 \mathrm{dpi}$, at which timepoint symptoms were first observed (Fig. 3). Moreover, accumulation of SBLV CP in uninoculated rosette leaves was similar among the three accessions (Fig. 3). Therefore, we concluded that Col-0, S96, and Ei-2 show different sensitivities to SBLV infection because of a mechanism that is independent of the efficiency of SBLV multiplication in plants.

\section{Genetic traits of symptom phenotypes.}

To determine the genetic basis of the symptom phenotypes in SBLV infection, we crossed Col-0 and S96 plants. All $F_{1}$ plants from the reciprocal crosses between Col-0 and S96 developed symptoms intermediate to those of the two parental accessions (Table 2). Necrotic symptoms were observed on inoculated leaves of the $\mathrm{F}_{1}$ plants and were like those of parental S96 at 10 dpi but were mild on uninoculated leaves. The curling of leaves and stems in uninoculated tissues was also mild at 14 dpi (Fig. 1B), and $F_{1}$ individuals produced seeds (data not shown). These results indicate that the symptom phenotype of S96 is conferred by a semidominant trait. The $F_{1}$ plants were self-pollinated, and the resulting $F_{2}$ plants were inoculated with SBLV to determine the segregation of the symptom phenotype of $S 96$. In $212 \mathrm{~F}_{2}$ individuals from the $\mathrm{F}_{1}$ population ( $\left.9 \mathrm{~S} 96 \times{ }^{\lambda} \mathrm{Col}-0\right)$ inoculated with $\mathrm{SBLV}$, severe symptoms were observed in 48 plants, mild symptoms were observed in 110 plants, and 54 plants were symptomless (Table 2). This segregation ratio of severe symptoms/mild symptoms/no symptoms was $0.9: 2: 1\left(\chi^{2}=0.64\right)$, which was similar to the expected ratio of $1: 2: 1$. In an additional analysis using $\mathrm{F}_{2}$ plants from the $\mathrm{F}_{1}$ population $\left(+\mathrm{Col}-0 \times{ }^{2} \mathrm{~S} 96\right)$, a similar segregation ratio was observed (Table 2). These results indicate that the symptom phenotype of S96 was conditioned by a single nuclear locus; this locus was designated SSB1 (symptom development by SBLV infection).

\section{Mapping of the SSB1 locus.}

The map position of the SSBI locus was determined by using the $\mathrm{F}_{2}$ population from the $\mathrm{F}_{1}$ plants $(q \mathrm{~S} 96 \times \delta \mathrm{Col}-0)$. Because it was difficult to extract genomic DNA from the plants showing severe symptoms, we selected mapping lines that did not show any symptoms of SBLV infection (Col-0 phenotype). The genotype at PCR-based DNA markers covering the $A$. thaliana genome was then determined. Nine markers displayed polymorphisms between Col-0 and S96 (ADH, AG, DHS1, GPA1.1, LFY3, nga6, nga8, nga225, and nga280). Initial mapping using these markers showed that two markers, AG and DHS1, which are located on chromosome IV, linked to the Col-0 phenotype (recombination frequency of $\mathrm{AG}$ and DHS1 were 16.3 and $4.9 \%$, respectively). The other markers showed no significant linkage with the Col-0 phenotype (re- 
combination frequency 45 to $65 \%$ ). Further mapping was performed using $76 \mathrm{~F}_{2}$ plants showing no symptoms. As shown in Figure 4, some markers on chromosome IV, especially CAT2, showed linkage with the SSBI locus. These data suggest that the SSB1 locus is located within a region between markers ATMYB3R (map position $82.27 \mathrm{cM}$ ) and nga1107 (map position $104.73 \mathrm{cM}$ ) on chromosome IV.
To examine whether the symptoms induced in Ei-2 were also controlled by the SSB1 locus, Ei-2 plants were crossed with Col- 0 or S96. $F_{1}$ plants from crosses between Ei-2 and Col-0 and between $\mathrm{Ei}-2$ and S96 were late flowering like Ei-2. When $\mathrm{F}_{1}$ plants from the cross between Ei-2 and Col-0 were inoculated with SBLV at 4 weeks after sowing, mild stunting was observed at $14 \mathrm{dpi}$ (Table 2), demonstrating that the symptoms
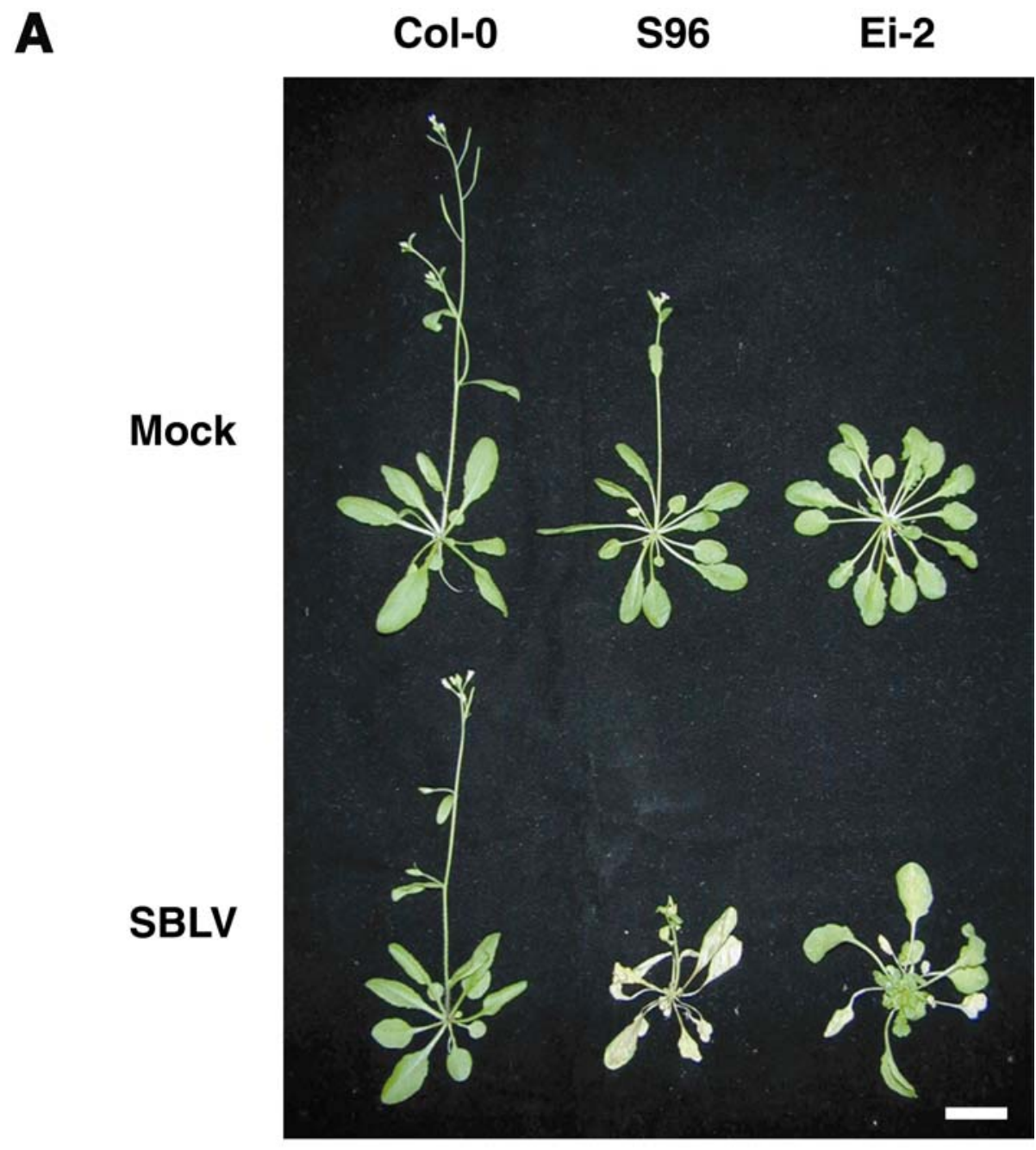

B

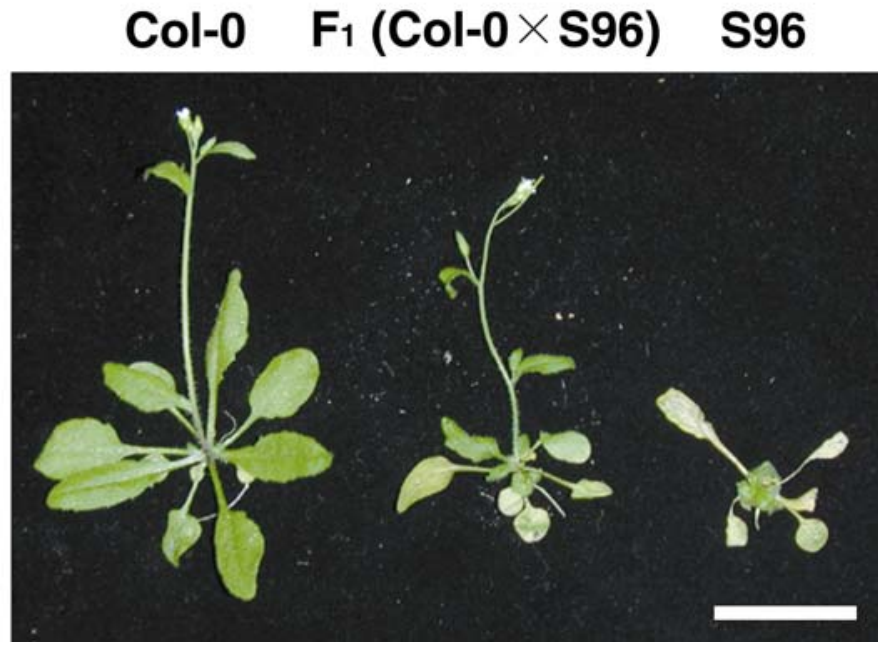

Fig. 1. Arabidopsis accessions showing symptoms induced by Spring beauty latent virus (SBLV) infection. A, Col-0, S96, and Ei-2 or B, $\mathrm{F}_{1}$ plants from the cross between S96 and Col-0 were inoculated with SBLV virions and were photographed at 14 days postinoculation. In A, Col-0, S96, and Ei-2 plants inoculated with buffer (upper) or SBLV (lower) are shown from left to right. Bar $=20 \mathrm{~mm}$. 
of Ei-2 are also conferred by a semidominant trait like those in S96. However, all $F_{1}$ plants from the cross between Ei-2 and S96 showed severe stunting symptoms like those of the parental Ei-2 (Table 2), indicating that the SSB1 locus of S96 functioned for development of severe symptoms in Ei-2. Furthermore, the symptom traits of Ei-2 showed linkage to a marker DHS1 that is located near the SSB1 locus (recombination frequency $7.1 \%$ ) in

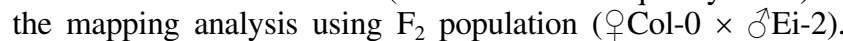
These data suggest that allelic SSB1 in Ei-2 is also involved in symptom development by SBLV infection.
Involvement of salicylic acid in necrotic symptoms of S96.

Salicylic acid (SA) is a key factor for plant defense responses to pathogens and is intimately associated with cell death (Overmyer et al. 2003). To examine whether SA is involved in symptom development in $A$. thaliana by SBLV infection, we tested the effect of the $N a h G$ gene encoding salicylate hydroxylase on necrotic symptoms induced in S96. We crossed S96 with either NahG transgenic plants (background: accession Nossen) or nontransgenic Nossen plants (No-0) and inoculated $F_{1}$ plants from these crosses with SBLV RNA,

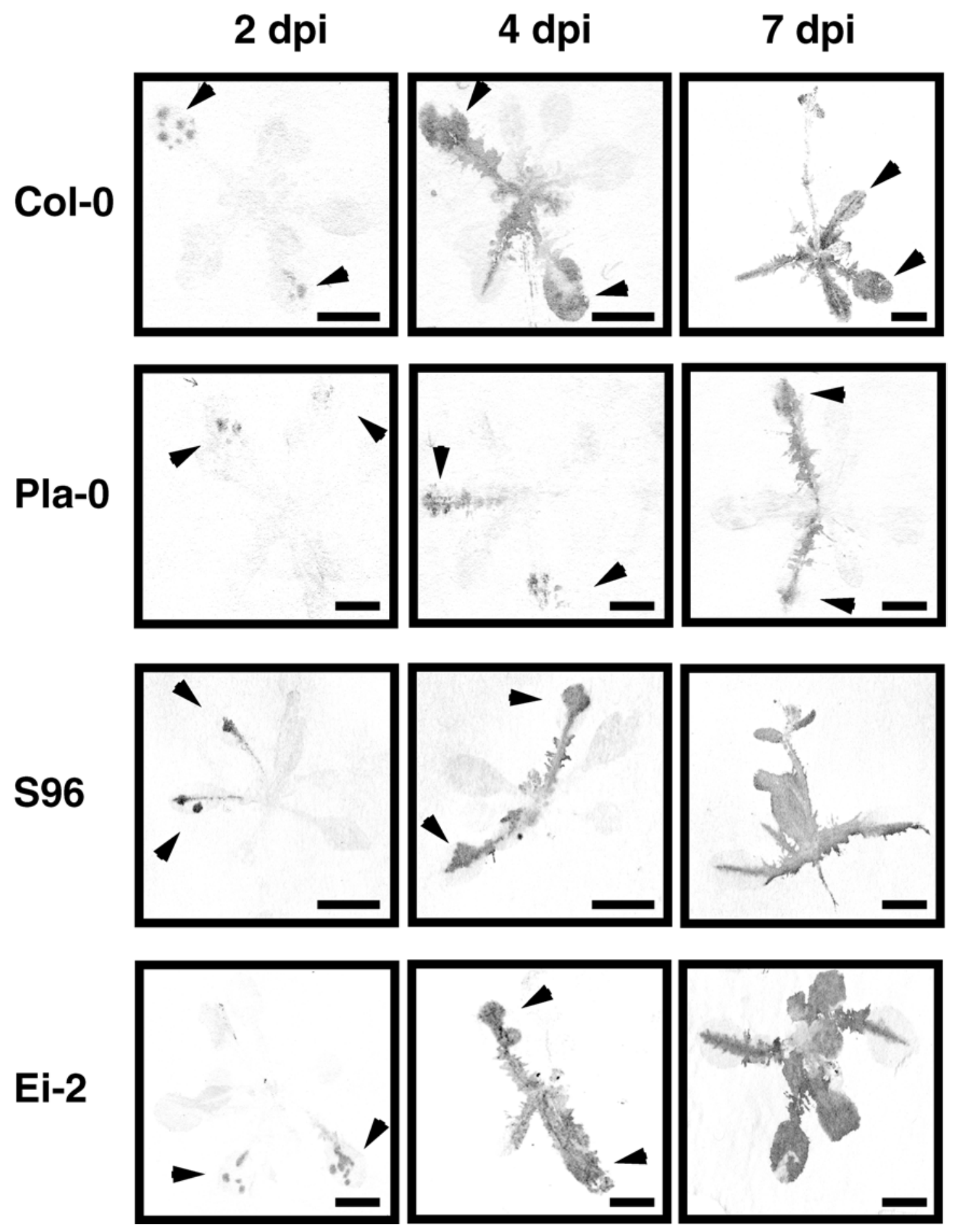

Fig. 2. Time-course for Spring beauty latent virus (SBLV) spread in Col-0, Pla-0, S96, and Ei-2. Plants were inoculated with SBLV virion and pressed onto chromatography papers at 2, 4, and 7 days postinoculation (dpi). The distribution of SBLV coat protein was immunologically detected. Arrowheads indicate inoculated leaves. Because inoculated leaves of S96 and Ei-2 were dead and desiccated at 7 dpi, these leaves are not shown. Bar $=10 \mathrm{~mm}$. 
which was used as inoculum instead of SBLV virion to avoid detecting input $\mathrm{CP}$ in press blot assay. Parental No-0 and $N a h G$ plants showed no visible symptoms of SBLV infection on either inoculated or uninoculated leaves, in contrast to S96
(Table 1 and Fig. 5A). Symptoms induced in $\mathrm{F}_{1}$ plants from the cross between S96 and No-0 were intermediate compared with those in the two parental accessions (data not shown), as were those of $\mathrm{F}_{1}$ plants from the cross between S96 and Col-0,
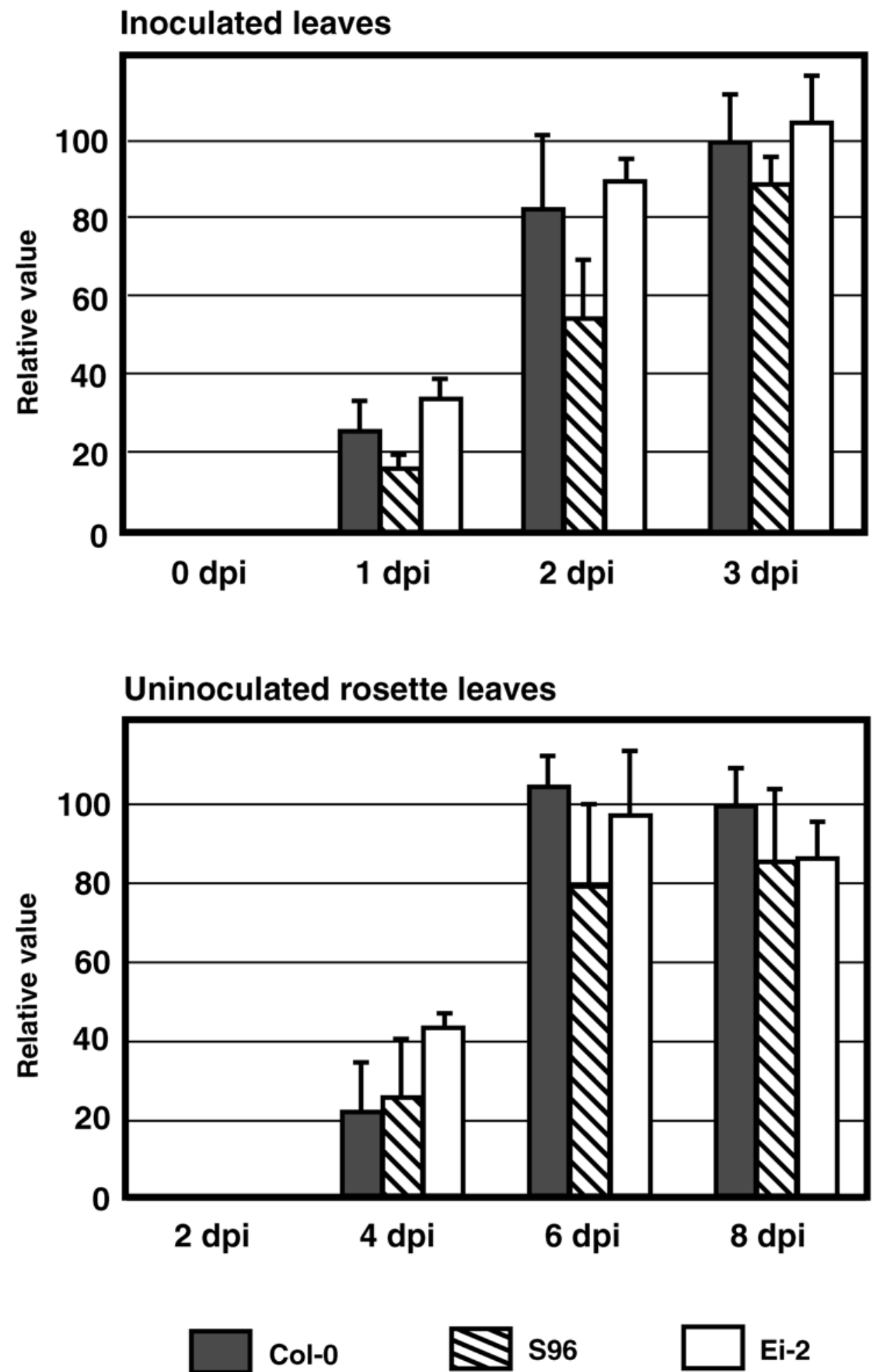

Fig. 3. Time-course of spring beauty latent virus (SBLV) coat protein (CP) accumulation in Col-0, S96, and Ei-2 plants. Two expanded leaves were inoculated with SBLV transcripts at 3 to 4 weeks after sowing. Inoculated and uninoculated rosette leaves were harvested at 0 , 1, 2, and 3 days postinoculation (dpi) and 2, 4, 6, and $8 \mathrm{dpi}$, respectively. The accumulation levels of SBLV CP were analyzed by Western blotting and then densitometrically quantified with the NIH Image program. The SBLV CP accumulations in inoculated (upper graph) and uninoculated (lower graph) rosette leaves of Col-0, S96, and Ei-2 are shown as closed, hatched, and open columns, respectively. Relative values for the accumulation of SBLV CP in leaves compared with those in inoculated leaves of Col-0 at 3 dpi (upper graph) or in uninoculated rosette leaves of Col-0 at 8 dpi (lower graph) are indicated. The mean values and standard deviations (vertical lines) were calculated from six to eight samples. Symptoms in S96 and Ei-2 were so severe after 3 dpi in inoculated leaves and after 8 dpi in uninoculated rosette leaves that accumulation of SBLV CP was not examined afterwards. 


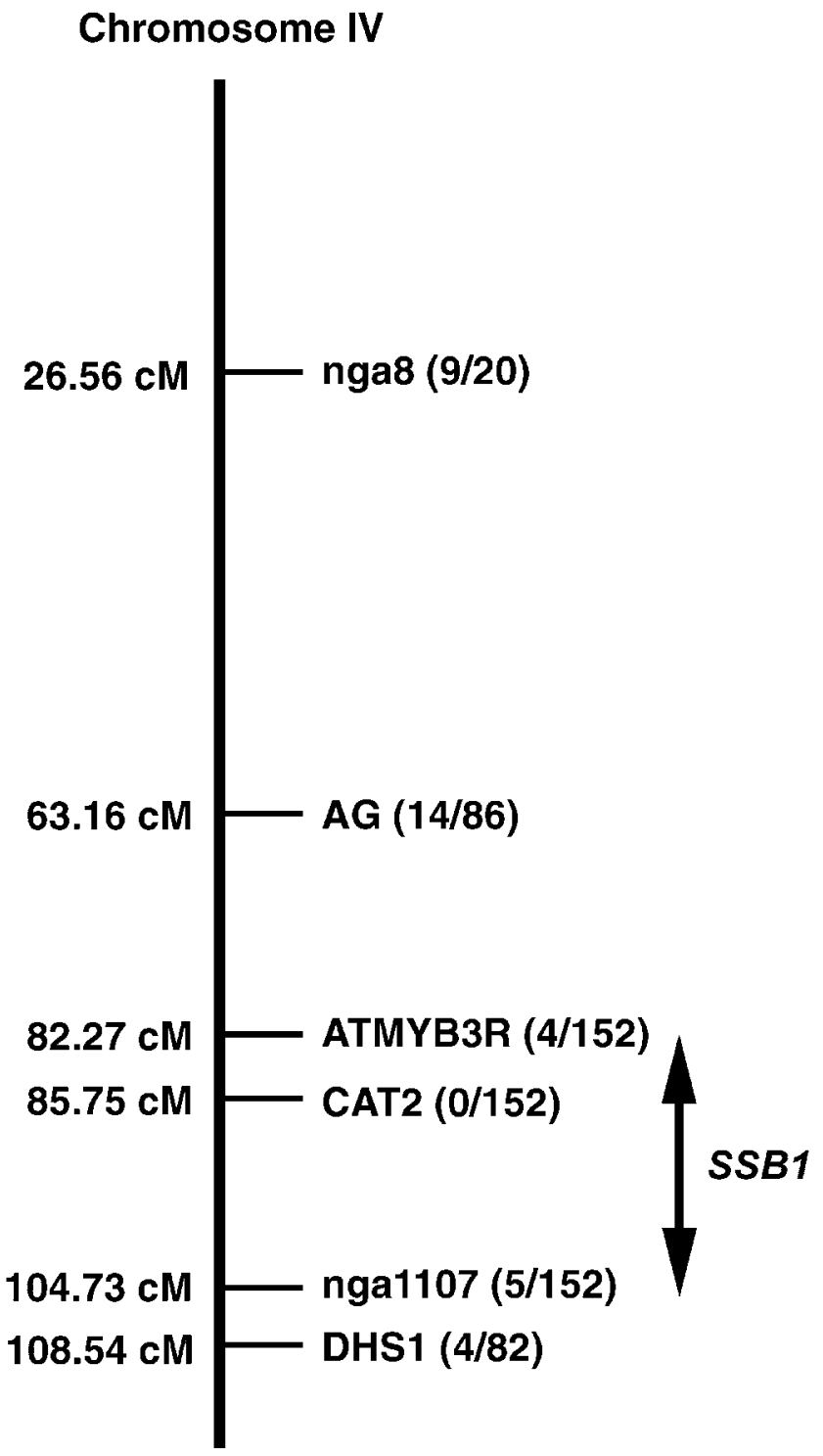

Fig. 4. Map position of SSB1 on chromosome IV in Arabidopsis thaliana. The map positions of the markers (the Arabidopsis Information Resource) are indicated at left. The markers on chromosome IV that were used for linkage analysis are indicated on the right. The number of chromatids recombined between a marker and the SSB1 locus with respect to the total number of chromatids examined is shown in parentheses. and inoculated leaves of $\mathrm{F}_{1}$ plants $(\mathrm{S} 96 \times \mathrm{No}-0)$ had died by 10 dpi (Fig. 5A). When $\mathrm{F}_{1}$ plants from the cross between $\mathrm{S} 96$ and $N a h G$ were inoculated with SBLV RNA, inoculated leaves of the $\mathrm{F}_{1}$ plants $(\mathrm{S} 96 \times N a h G)$ exhibited mild yellowing and small necrotic lesions at $10 \mathrm{dpi}$ but, in contrast to $\mathrm{F}_{1}$ plants $(\mathrm{S} 96 \times$ No-0), did not die (Fig. 5A). Even when symptoms were observed at $20 \mathrm{dpi}$, necrotic symptoms did not expand (data not shown). Press blot assays demonstrated that SBLV spread throughout the inoculated leaves (Fig. 5B). These data strongly suggest that $\mathrm{SA}$ has a significant involvement in symptom development in S96 by SBLV infection.

\section{DISCUSSION}

In this study, we examined the response of 63 Arabidopsis accessions against SBLV infection as a model for analyzing plant-bromovirus interactions. Recently, precise infectivity of $\mathrm{BMV}$ in A. thaliana was reported, and BMV was shown to systemically infect Col-0 under a certain plant growth condition (Dzianott and Bujarski 2004), which suggests that BMV can be used to identify plant factors governing its systemic infection. On the other hand, the accumulation level of BMV RNA in uninoculated leaves of Col-0 was at least 500-fold lower than that in barley (Dzianott and Bujarski 2004). In our data, the accumulation level of SBLV CP was approximately $800 \mu \mathrm{g}$ per $\mathrm{g}$ of tissue in uninoculated rosette leaves of Col-0 at 14 dpi (F. Hagihara and K. Mise, unpublished data), which is fivefold lower than that of BMV CP in barley (Okinaka et al. 2001). Taken together, these data indicate that SBLV multiplication in Col-0 is more efficient than that of BMV. Therefore, SBLV may be more useful in some studies, such as the analysis of virus pathogenicity in plants, as reported in this article.

In most Arabidopsis accessions, including Col-0, SBLV infection does not induce visible symptoms, but severe symptoms are induced in some accessions, such as S96. Genetic analysis suggests that symptoms induced in S96 are conditioned by a single nuclear gene, $S S B 1$. It has been reported that natural diversity in response to pathogens often occurs in plant genes involved in the recognition of plant pathogens, for example, $R$ genes for gene-for-gene resistance (Cooley et al. 2000; Takahashi et al. 2002). Importantly, plant components functioning downstream of the plant defense responses are not diverse among accessions. Therefore, the SSB1 gene product of S96 but not of Col-0 may be involved in the recognition of SBLV components and may activate plant responses to develop necrotic symptoms, as do $R$ genes. Another possibility is that allelic SSBI in Col-0 is functional and confers tolerance to SBLV infection. In this model, recognition of SBLV in A. thaliana for symptom induction may be

Table 2. Genetic analysis of symptoms in response to Spring beauty latent virus (SBLV) infection

\begin{tabular}{|c|c|c|c|c|c|}
\hline \multirow[b]{2}{*}{ Cross } & \multicolumn{3}{|c|}{ Symptoms ${ }^{a}$} & \multirow[b]{2}{*}{$\chi^{2 b}$} & \multirow[b]{2}{*}{$P^{\mathrm{c}}$} \\
\hline & Severe & Mild & None & & \\
\hline 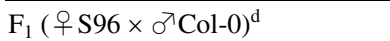 & 0 & 21 & 0 & - & - \\
\hline 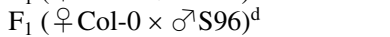 & 0 & 18 & 0 & - & - \\
\hline $\mathrm{F}_{2}\left(\text { selfed } \mathrm{F}_{1}\left[\text { 우 } \mathrm{S} 96 \times \sigma^{\top} \mathrm{Col}-0\right]\right)^{\mathrm{e}}$ & 48 & 110 & 54 & 0.64 & 0.73 \\
\hline $\mathrm{F}_{2}\left(\text { selfed } \mathrm{F}_{1}\left[\text { 우 Col- } 0 \times \sigma^{\top} \mathrm{S} 96\right]\right)^{\mathrm{e}}$ & 24 & 50 & 20 & 0.72 & 0.70 \\
\hline $\mathrm{F}_{1}\left(\text { 우 Col- } 0 \times \sigma^{\urcorner} \mathrm{Ei}-2\right)^{\mathrm{f}}$ & 0 & 10 & 0 & - & - \\
\hline 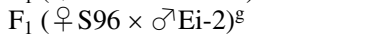 & 11 & 0 & 0 & - & - \\
\hline
\end{tabular}

a Plants were inoculated with the SBLV virion and scored for symptoms at 14 dpi.

${ }^{\mathrm{b}} \chi^{2}$ values were calculated for an expected ratio of $1: 2: 1$ for severe/mild/no symptoms in plants.

${ }^{\mathrm{c}}$ Probability ( $P$ value) was calculated using GraphPad Quickcalc online calculator.

${ }^{\mathrm{d}} \mathrm{F}_{1}$ plants from the reciprocal crosses between $\mathrm{S} 96$ (severe) and Col-0 (none) developed symptoms intermediate to those between the two parental accessions.

${ }^{\mathrm{e}} \mathrm{F}_{2}$ plants were obtained by allowing $\mathrm{F}_{1}$ individuals to self-fertilize.

${ }^{\mathrm{f}} \mathrm{F}_{1}$ plants from crosses between Ei-2 (severe) and Col-0 (none) developed mild stunting symptoms.

${ }^{\mathrm{g}} \mathrm{F}_{1}$ plants from crosses between $\mathrm{S} 96$ and Ei-2 showed systemic stunting symptoms like parental Ei-2. 
independent of $S S B 1$, and $S S B 1$ may repress the activation of plant responses involved in symptom development, for example, by interfering with the SBLV recognition. In addition, we cannot rule out the possibility that the $S S B I$ genes in both S96 and Col-0 are involved in normal development and maintenance of living cells in A. thaliana, and SBLV may specifically interfere with the function of SSB1 in S96. Because the $\mathrm{S} 96$ phenotype is semidominant, we cannot postulate whether $S S B 1$ functions to develop symptoms in S96 or to confer tolerance in Col-0.
In this study, the $N a h G$ gene critically affected development of necrotic symptoms, suggesting that SA plays a crucial role in symptom production in S96. In the case of a bacterial pathogen, it has been shown that plant hormones such as SA are essential for the production of disease symptoms in $A$. thaliana (O'Donnell et al. 2003). Moreover, SA is associated with leaf senescence and HR formation in gene-for-gene resistance, which are regarded as programmed cell death (PCD) (Overmyer et al. 2003; Yoshida 2003). Considering these reports, SBLV in S96 may activate the SA signaling pathway

A

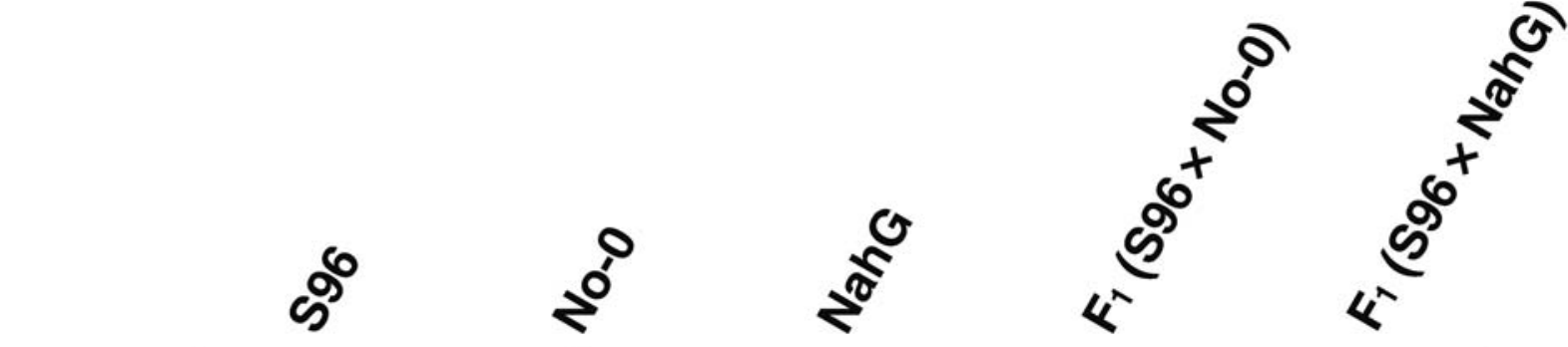

Mock

SBLV

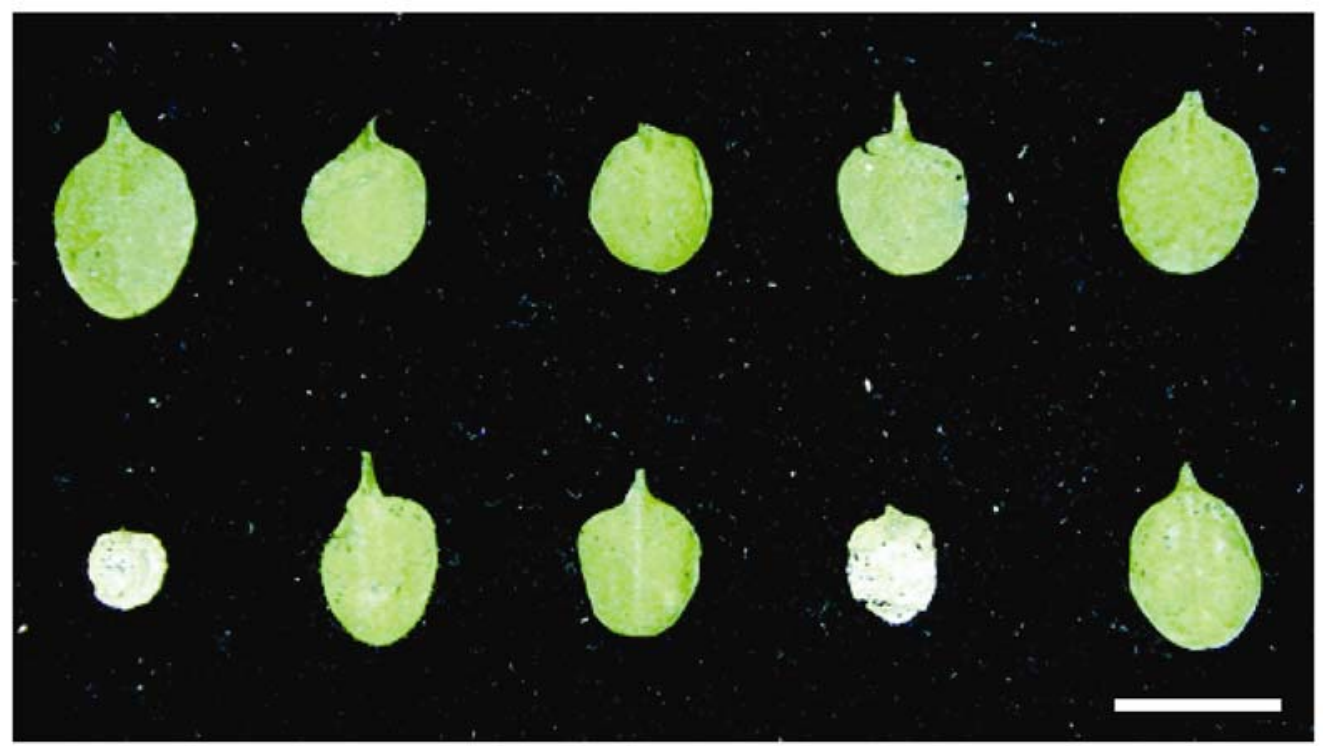

B

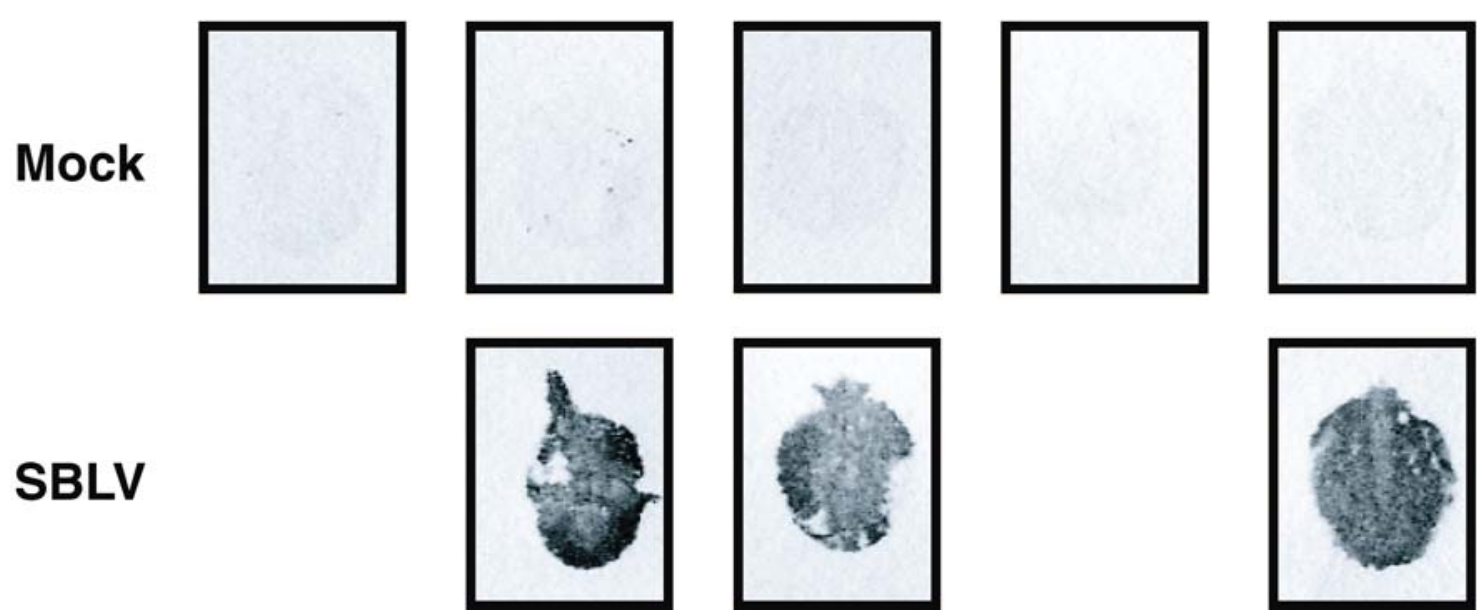

Fig. 5. Necrotic symptoms in inoculated leaves of $\mathrm{F}_{1}$ plants from crosses between $\mathrm{S} 96$ and No-0 and between S96 and NahG transgenic plants. A, Plants were inoculated with spring beauty latent virus (SBLV) RNA and were photographed at 10 dpi. B, Then SBLV coat protein distribution in the leaves was checked immunologically by press blot analysis. Mock-inoculated (upper) and SBLV-inoculated (lower) leaves are shown in each panel. Inoculated leaves

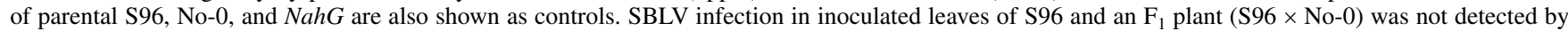
press blot assay, because the leaves were dead and desiccated. $\mathrm{Bar}=10 \mathrm{~mm}$. 
and induce PCD. Although the NahG gene clearly interfered with symptom production in $\mathrm{F}_{1}$ plants from the cross between S96 and NahG plants, the $\mathrm{F}_{1}$ plants showed mild yellowing and small necrotic lesions, indicating that symptom development was incompletely inhibited in this generation. This may imply that other pathways independent of SA signaling are also involved in symptom development, although there remains a possibility of unexpected effects from using the $F_{1}$ generation, for example, heterozygosity of the NahG gene. Further analyses of the effects of mutation in the components involved in SA-dependent signaling and other pathways, such as jasmonic acid- and ethylene-dependent signaling, on symptom production will facilitate understanding the mechanisms of symptom development in the SBLV-S96 system.

Multiplication of SBLV in S96 was similar to that in Col-0, indicating that SSBI controls symptom development in $A$. thaliana but not SBLV infectivity. A previous study has demonstrated that TRSV systemically induced necrotic symptoms in Estland but does not induce visible symptoms in Col-0. In that study, Lee and associates (1996) reported that the TTRI locus, which controls responses to TRSV, does not have significant effects on virus multiplication. Similar responses to SBLV and TRSV in A. thaliana suggest that the SSBI and TTRI loci control responses to viruses via similar mechanisms although, because the TTRl locus is located on chromosome $\mathrm{V}$, it is not allelic to SSB1. Identification of TTRI and SSBI genes will contribute to the elucidation of a mechanism that determines symptom development in plants by virus infection.

\section{MATERIALS AND METHODS}

Plants, viruses, and virus inoculation.

Seeds of $A$. thaliana accessions were obtained from the Arabidopsis Biological Resource Center, Ohio State University (Columbus, OH, U.S.A.). Seeds of NahG transgenic A. thaliana (Shah et al. 1999) were provided by H. Takahashi. Seeds were sown on rockwool, were treated at $4{ }^{\circ} \mathrm{C}$ in the dark for 2 days, and then, were grown in a growth room at $25^{\circ} \mathrm{C}$ with $16 \mathrm{~h}$ of illumination per day in nutrient medium (Dhingra and Sinclair 1985). SBLV (ATCC PV-369, American Type Culture Collection) virion $(0.5 \mu \mathrm{g} / \mu \mathrm{l})$, virion RNA $(0.5 \mu \mathrm{g} / \mu \mathrm{l})$, and in vitro transcripts $(0.2 \mu \mathrm{g} / \mu \mathrm{l}$ each of RNAs 1,2 , and 3$)$ were prepared and inoculated into A. thaliana as described previously (Fujisaki et al. 2003a).

\section{Detection of SBLV infection.}

The press blot assay for detecting the distribution of SBLV $\mathrm{CP}$ in $A$. thaliana was performed as previously described (Fujisaki et al. 2003b; Takahashi et al. 2001). To analyze the accumulation of SBLV CP, infected leaves were ground in Laemmli's sample buffer (Laemmli 1970) and were subjected to sodium dodecyl sulfate-polyacrylamide gel electrophoresis (samples from $0.4 \mathrm{mg}$ of fresh weight tissues were loaded into each lane), followed by immunoblot analysis as described previously (Damayanti et al. 1999). In both assays, SBLV CP was detected using a rabbit anti-BMV antiserum (ATCC PVAS178). The accumulation of SBLV CP was densitometrically quantified using the NIH Image program (National Institutes of Health, Bethesda, MD, U.S.A.).

\section{Genetic analysis.}

To analyze the genetic basis of sensitivity to SBLV, S96 plants were crossed with Col-0, Ei-2, No-0, or NahG plants. $\mathrm{F}_{1}$ plants were self-fertilized to obtain $\mathrm{F}_{2}$ seeds, and symptoms of $F_{1}$ and $F_{2}$ plants induced by SBLV infection were observed at 10 or 14 dpi. Infection with SBLV was confirmed by press blot analysis. In addition, we crossed Ei- 2 with Col-0 $(+\mathrm{Col}-0 \times$ $\left.{ }^{7} \mathrm{Ei}-2\right) . \mathrm{F}_{1}$ plants from these crosses were inoculated with SBLV as described above.

\section{Mapping of the SSB1 locus.}

To map the SSB1 locus, we used 14 simple sequence-length polymorphism (SSLP) markers (nga63, nga248, nga280, nga168, nga6, nga112, nga8, nga225, nga139, nga76, nga1111, nga1139, nga1107, and DET1.2) and 17 cleaved amplified polymorphic sequence (CAPS) markers (NCC1, ADH, GPA1.1, GL1, AG, DHS1, ASA1, DFR1, LFY3, G4539, PG11, RPS2, PRHA, ATMYB3R, CAT2, UM596A, and CCR1). Information on these DNA markers was obtained from the Arabidopsis Information Resource database. Genomic DNA of A. thaliana was purified as described previously (Edwards et al. 1991). PCR was performed under the conditions described by Bell and Ecker (1994) for SSLP markers and those described by Konieczny and Ausubel (1993) for CAPS markers. Genotypes at these markers in S96 and Col-0 genomes were checked, and markers showing polymorphism between the two accessions

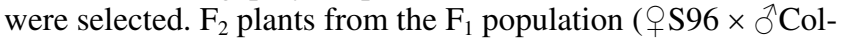
0 ) were inoculated with SBLV, and plants showing no visible symptoms at $14 \mathrm{dpi}$ were used for mapping. In initial mapping, we used nga63, nga280, nga6, nga112, nga8, nga225, nga139, NCC1, ADH, GPA1.1, AG, DHS1, and LFY3. Further mapping of the SSB1 locus on chromosome IV was performed using nga1139, nga1107, ATMYB3R, and CAT2.

\section{ACKNOWLEDGMENTS}

We thank H. Takahashi for Arabidopsis seeds of NahG transgenic plants, and M. Yoshii, S. Takeda, M. Ishikawa, and K. Okada for technical assistance. This work was supported in part by a grant-in-aid (12052201) for Scientific Research on Priority Area (A) from the Ministry of Education, Culture, Sports, Science and Technology, Japan, a grant-in-aid (15380035) for Scientific Research (B), and a grant-in-aid (13306005) for Scientific Research (A) from the Japan Society for the Promotion of Science.

\section{LITERATURE CITED}

Ahlquist, P. 1999. BROMOVIRUSES (BROMOVIRIDAE). Pages 198-204 in: Encyclopedia of Virology, 2nd ed. Vol. 1. A. Granoff and R. G. Webster. eds. Academic Press, San Diego, CA, U.S.A.

Ahlquist, P., French, R., Janda, M., and Loesch-Fries, L. S. 1984. Multicomponent RNA plant virus infection derived from cloned viral cDNA Proc. Natl. Acad. Sci. U.S.A. 81:7066-7070.

Alonso-Blanco, C., and Koornneef, M. 2000. Naturally occurring variation in Arabidopsis: An underexploited resource for plant genetics. Trends Plant Sci. 5:1360-1385.

Bell, C. J., and Ecker, J. R. 1994. Assignment of 30 microsatellite loci to the linkage map of Arabidopsis. Genomics 19:137-144.

Chisholm, S. T., Mahajan, S. K., Whitham, S. A., Yamamoto, M. L., and Carrington, J. C. 2000. Cloning of the Arabidopsis RTM1 gene, which controls restriction of long-distance movement of Tobacco etch virus. Proc. Natl. Acad. Sci. U.S.A. 97:489-494.

Cooley, M. B., Pathirana, S., Wu, H. J., Kachroo, P., and Klessig, D. F. 2000. Members of the Arabidopsis HRT/RPPS family of resistance genes confer resistance to both viral and oomycete pathogens. Plant Cell 12:663-676

Damayanti, T. A., Nagano, H., Mise, K., Furusawa, I., and Okuno, T. 1999. Brome mosaic virus defective RNAs generated during infection of barley plants. J. Gen. Virol. 80:2511-2518.

Dardick, C. D., Golem, S., and Culver, J. N. 2000. Susceptibility and symptom development in Arabidopsis thaliana to Tobacco mosaic virus is influenced by virus cell-to-cell movement. Mol. Plant-Microbe Interact. 13:1139-1144.

Dempsey, D. A., Wobbe, K. K., and Klessig, D. F. 1993. Resistance and susceptible responses of Arabidopsis thaliana to Turnip crinkle virus. Phytopathology 83:1021-1029.

Dhingra O., and Sinclair, J. 1985. Basic Plant Pathology Methods. CRC Press, Boca Raton, FL, U.S.A.

Dzianott, A. M., and Bujarski, J. J. 2004. Infection and RNA recombination of Brome mosaic virus in Arabidopsis thaliana. Virology 318:482-492. 
Eckardt, N. A. 2001. Arabidopsis genome conference 2000. How a small weed changed the world. Plant Cell 13:5-10.

Eckardt, N. A., Araki, T., Benning, C., Cubas, P., Goodrich, J., Jacobsen, S. E., Masson, P., Nambara, E., Simon, R., Somerville, S., and Wasteneys, G. 2001. Arabidopsis research 2001. Plant Cell 13:1973-1982.

Edwards, K., Johnstone, C., and Thompson, C. 1991. A simple and rapid method for the preparation of plant genomic DNA for PCR analysis. Nucleic Acids Res. 19:1349.

Fujisaki, K., Hagihara, F., Kaido, M., Mise K., and Okuno, T. 2003a. Complete nucleotide sequence of Spring beauty latent virus, a bromovirus infectious to Arabidopsis thaliana. Arch. Virol. 148:165-175.

Fujisaki, K., Kaido, M., Mise, K., and Okuno, T. 2003b. Use of Spring beauty latent virus to identify compatible interactions between bromoviurs components required for virus infection. J. Gen. Virol. 84:13671375.

Konieczny, A., and Ausubel, F. M. 1993. A procedure for mapping Arabidopsis mutations using co-dominant ecotype-specific PCR-based markers. Plant J. 4:403-410.

Kover, P. X., and Schaal, B. A. 2002. Genetic variation for disease resistance and tolerance among Arabidopsis thaliana accessions. Proc. Natl. Acad. Sci. U.S.A. 99:11270-11274.

Laemmli, U. K. 1970. Cleavage of structural proteins during the assembly of the head of bacteriophage T4. Nature 227:680-685.

Lane, L. C. 1981. Bromoviruses. Pages 333-376 in: Handbook of Plant Virus Infections and Comparative Diagnosis. E. Kurstak, ed. Elsevier Biomedical Press, Amsterdam.

Lee, S., Stenger, D. C., Bisaro, D. M., and Davis, K. R. 1994. Identification of loci in Arabidopsis that confer resistance to geminivirus infection. Plant J. 6:525-535.

Lee, J. M., Hartman, G. L., Domier, L. L., and Bent, A. F. 1996. Identification and map location of TTR1, a single locus in Arabidopsis thaliana that confers tolerance to tobacco ringspot nepovirus. Mol. PlantMicrobe Interact. 9:729-735.

Leisner, S. M., and Howell, S. H. 1992. Symptom variation in different Arabidopsis thaliana ecotypes produced by Cauliflower mosaic virus. Phytopathology, 82:1042-1046.

Martín Martín, A., Cabrera y Poch, H. L., Martínez-Herrera, D, , and Ponz, F. 1999. Resistance to turnip mosaic potyvirus in Arabidopsis thaliana. Mol. Plant-Microbe Interact. 12:1016-1021.

O'Donnell, P. J., Schmelz E. A., Moussatche, P., Lund, S. T., Jones, J. B. and Klee, H. J. 2003. Susceptible to intolerance-A range of hormonal actions in a susceptible Arabidopsis pathogen response. Plant J. 33:245-257

Okinaka, Y., Mise, K., Suzuki, E., Okuno, T., and Furusawa, I. 2001. The $\mathrm{C}$ terminus of brome mosaic virus coat protein controls viral cell-to-cell and long-distance movement. J. Virol. 75:5385-5390.
Overmyer, K., Brosche M., and Kangasjarvi, J. 2003. Reactive oxygen species and hormonal control of cell death. Trends Plant Sci. 8:335-342.

Park, S. H., Hur J., Park J., Lee, S., Lee T. K., Chang M., Davi, K. R., Kim, J., and Lee, S. 2002. Identification of a tolerant locus on Arabidopsis thaliana to hypervirulent beet curly top virus CFH strain. Mol. Cells 13:252-258.

Revers, F., Guiraud, T., Houvenaghel, M. C., Mauduit, T., Le Gall, O., and Candresse, T. 2003. Multiple resistance phenotypes to Lettuce mosaic virus among Arabidopsis thaliana accessions. Mol. Plant-Microbe Interact. 16:608-616.

Shah, J., Kachroo, P., and Klessig, D. F. 1999. The Arabidopsis ssil mutation restores pathogenesis-related gene expression in $n p r l$ plants and renders defensin gene expression salicylic acid dependent. Plant Cell 11:191-206.

Simon, A. E., Li, X. H., Lew, J. E., Stange, R., Zhang, C., Polacco, M., and Clifford, D. 1992. Susceptibility and resistance of Arabidopsis thaliana to Turnip crinkle virus. Mol. Plant-Microbe Interact. 5:496-503.

Sullivan, M. L., and Ahlquist, P. 1997. cis-Acting signals in bromovirus RNA replication and gene expression: Net-working with viral proteins and host factors. Semin. Virol. 8:221-230.

Takahashi, H., Goto, N., and Ehara, Y. 1994. Hypersensitive response in cucumber mosaic virus-inoculated Arabidopsis thaliana. Plant J. 6:369377.

Takahashi, H., Suzuki, M., Natsuaki, K., Shigyo, T., Hino, K., Teraoka, T., Hosokawa, D., and Ehara, Y. 2001. Mapping the virus and host genes involved in the resistance response in cucumber mosaic virus-infected Arabidopsis thaliana. Plant Cell Physiol. 42:340-347.

Takahashi, H., Miller, J., Nozaki, Y., Sukamuto, Takeda, M., Shah, J., Hase, S., Ikegami, M., Ehara, Y., and Dinesh-Kumar, S. P. 2002. RCY1, an Arabidopsis thaliana RPP8/HRT family resistance gene, conferring resistance to Cucumber mosaic virus requires salicylic acid, ethylene and a novel signal transduction mechanism. Plant J. 32:655-667.

Yoshida, S. 2003. Molecular regulation of leaf senescense. Curr. Opin. Plant Biol. 6:79-84.

Whitham, S. A., Anderberg, R. J., Chisholm, S. T., and Carrington J. C. 2000. Arabidopsis RTM2 gene is necessary for specific restriction of Tobacco etch virus and encodes an unusual small heat shock-like protein. Plant Cell 12:569-582.

\section{AUTHOR-RECOMMENDED INTERNET RESOURCES}

The Arabidopsis Information Resource (TAIR) database: www.arabidopsis.org/

GraphPad Quickcalcs online calculators: www.graphpad.com/quickcalcs/index.cfm 